\title{
Hierarchical Wigner Crystal at the Edge of Quantum Hall Bar
}

\author{
Yuli V. Nazarov \\ Faculteit der Technische Natuurkunde, Technische Universiteit Delft, 2628 CJ Delft, the \\ Netherlands
}

\begin{abstract}
We show that quasiholes persist near the edge of incompressible Quantum Hall state forming a Wigner structure. The average density of quasiholes is fixed by electrostatics and decreases slowly with increasing distance from the edge. As we see from elementary reasoning, their specific arrangement can not be a regular Wigner lattice and shows a complex hierarchical structure of dislocations.
\end{abstract}

73.20.Dx 71.45.Gm 71.50.+t

Typeset using REVTEX 
It is known that a homogeneous 2D electron gas in magnetic field may form an incompressible state if the electron density $n$ satisfies the following magic relation: $n=p / q H / \Phi_{0}$, $p, q$ being integer numbers, $\Phi_{0}=2 \pi \hbar c / e$. This is a physical origin of Integer and Fractional Quantum Hall effects. [1]

In reality, 2D gas structures are finite. Their size is usually controlled by means of metal gate electrodes imposed on the top of the heterostructure and biased at large negative potential. The electrodes deplete the $2 \mathrm{D}$ underneath and thus shape it. $2 \mathrm{D}$ gas is not homogeneous near the edge. The structure of the edge of the incompressible state always receives much attention. [2] The origin of this is that the electric transport is believed to take place only near the edge. Early studies neglected interelectron electrostatic interactions. In this approach, the edge of incompressible state is very sharp being smoothed at distances of the order of magnetic length $l_{H} \equiv \sqrt{\hbar c / e H}$. Owing to the magic relation, $l_{H}$ is of the order of interelectron distance. Lately it has been recognized that the macroscopic electrostatics is of a crucial importance for the problem. Powerful electric forces dilute the 2D near the edge at distances much larger than $l_{H}$. Moreover, a small deficit of the electron density persists far from the edge. This concept was first introduced in [3] and has been given a refined formulation in [4].

In present study we concentrate on the region where the deficit of the electron density is small compared to $n$. We argue that the deficit is due to discrete quasiholes. We regard those as classical particles with Coulomb interaction. The arrangement of the quasiholes must be close to Wigner crystal. However, it is not a regular lattice. We argue that the actual arrangement is a hierarchical crystal of dislocations in Wigner lattice. The arguments given are very straightforward and appear to be of a general nature.

Let us first recall the electrostatic picture of the Hall bar edge. [4] We restrict ourselves to a simplest geometry (Fig.1). 2D gas and the gate electrode are assumed to be in the same plane $z=0$, the density and voltage distribution are uniform in $y$ direction. The gate electrode is at $x<0$, the $2 \mathrm{D}$ gas density reaches is unperturbed value $n(\infty)$ at $x \rightarrow$ $\infty$. Let $a$ be a separation between $2 \mathrm{D}$ gas and the gate. The density profile is given by $n(x)=n(\infty) \sqrt{\frac{x-a}{x}}$. The separation is determined by the voltage difference $V_{g}$ between the gate and the $2 \mathrm{D}$ gas, $\left.a=V_{g} \epsilon\left(2 \pi^{2} n(\infty) e\right)^{-1}\right) . \epsilon>>1$ being the dielectric constant of the host material. As it has been argued in [4, this density profile remains practically unchanged if one takes into account the inner properties of $2 \mathrm{D}$ gas. The reason is that the inner energies characterizing the 2D gas such as Fermi energy, Landau level separation, characteristic Coulomb energy $e^{2} \sqrt{n} / \epsilon$ are much smaller than the gate voltage $V_{g}$. Thus nothing in 2D can withstand the external electrostatic forces and provide sensible deviations from the profile.

Let us consider an asymptotic region $x>>a$. In this region the density deficit has a long power-law tail

$$
n(\infty)-n(x)=\frac{a}{2 x} \equiv \frac{V_{g} \epsilon}{4 \pi^{2} e x}
$$

We can verify this peculiar shape of the tail considering the electrostatic problem simplified to a primary school level. Let us consider it at the scales larger than $a$. The potential distribution can be readily found from boundary conditions $V(x)=0$ at $x>0, V(x)=$ $V_{g}$ at $x<0$ if $z=0$. The equipotentials are just rays beginning in the origin, and $V(z, x)=$ $2 V_{g} / \pi|\arctan (x / z)|$. Derivative of $V(x, z)$ with respect to $z$ at $z=0, x>0$ gives an electric 
field perpendicular to 2D plane and, consequently, the density deficit induced by the gate. This, of course, coincides with (11).

If $n(\infty)$ corresponds to a metallic, compressible state there is no difficulty to change its density and provide a small density deficit. Very different situation takes place if $n(\infty)$ corresponds to an incompressible state satisfying the magic relation. By virtue of incompressibility, the density deficit must be dumped into positively charged excitations on the background of the incompressible state(quasiholes). The nature of these excitations depends on the type of incompressible state: for IQHE, those are just common holes with elementary charge. For FQHE incompressible state, the quasiholes bear a fractional charge $e / q$. Electrostatic interaction between holes can not be screened by the incompressible background, so they repel each other as common charges do. As common charges, the quasiholes are affected by magnetic field that localizes their wave functions at space scale of the order of $l_{H}$. If the density deficit is small enough, the average distance between quasiholes is much larger than magnetic length. Due to repulsion, they never come close to each other, their wave function do not overlap and quantum mechanical effects are negligible. Therefore at low densities the quasiholes can be viewed as classical particles subjected to Coulomb repulsion, exactly like electrons forming the common Wigner structure. [5] Very similar situation in quantum dots has been studied in [6].

At larger densities quasihole wave functions are forced to overlap and they form a collective metallic state. So far no theory gives a quantitative description of this transition. To estimate the critical concentration, one can draw an analogy with the low density electron gas where such transition occurs at $n_{c} \approx 0.2 H / \Phi_{0}$. [1] Summarizing all that, we shall accept the following picture of the Quantum Hall bar edge (Fig. 1): the area adjacent to the edge can be schematically separated into two regions. In the region I, the density deficit is big enough to provide the metallic state. This region may be subdivided into several disconnected metallic regions by dipolar strips corresponding to incompressible states with density lower than $n$. [4] In the region II, we have the incompressible state doped by distinct quasiholes. The density of dopants drops according to Eq. 1 as $n_{0} \equiv q(n(\infty)-n(x))=\frac{V_{g} q \epsilon}{4 \pi^{2} e x} \equiv \frac{1}{b x}$. A good estimation for $a, b$ would be $b=3 \mathrm{~nm}, a=400 \mathrm{~nm}$.

Now we are in position to analyze what is the equilibrium arrangement of the quasiholes far in the region II, at $x>>a$. In the following consideration we shall completely neglect the potential disorder. It may be very important in the systems of the kind since the quasiparticles can be pinned in random potential minima. In a realistic structure, random potential landscape is created by donors spatially separated from 2D gas. The Wigner structure survives provided the separation exceeds the average distance between (quasi)particles. Since there are no fundamental reasons limiting the separation the structures may be in principle as clean as required. Anyway, it seems to be logical to comprehend first the situation in the absence of disorder.

Let us recall that we now deal with classical particles. They repel each other and their average density is fixed by external means. If the fixed average density were uniform the particles would form a triangular Wigner lattice. We shall expect that their local arrangement is close to the one if the density varies slowly. However, it is easy to see that the global arrangement can not be a regular lattice of any kind. The only way to change the density in a regular lattice is to stretch it in $x$ direction. This procedure does not preserve the local triangular arrangement of the particles and the resulting stricture would have been 
extremely energy unfavorable. In terms of elasticity theory, [7] there would be an enormous stress in the lattice. There should be some defects in the lattice to relieve the stress and to restore the favorable local arrangement.

The minimal energy configuration is provided by point-like defects: dislocations. The triangular local arrangement will be distorted for the particles only within several lattice spacings from a dislocation and will be preserved for the most of them. The density of dislocations can be evaluated from the following consideration. Let us suppose that the local triangular arrangement is realized for most of the particles. The number of rows (extended in $x$ direction) per unit $y$ length is then given by $N_{\text {row }}=\beta \sqrt{n_{0}}, \beta$ being lattice-dependent dimensionless coefficient, $\beta=2 / 3^{3 / 4}$ for triangular lattice. Each dislocation is known to swallow one row [7], so that the dislocation density is just a derivative of the number of rows with respect to $x$ :

$$
n_{1}=-\frac{d}{d x} N_{\text {row }}=-\beta \frac{d}{d x} \sqrt{n_{0}}=\frac{\beta}{2 \sqrt{b x^{3}}}
$$

We see that $n_{1}<<n_{0}$ in the region of interest, this validates the scheme we use.

We seem to advance in understanding of particle arrangement. The question remains what is the dislocation arrangement. They also can be viewed as particles, and their average density is fixed by the relation (2). As we know from elasticity theory, [7] they repel each other. So we come back to the initial problem with changed fixed density! We have to repeat the procedure considering dislocations in the lattice of dislocations, dislocations in the lattice of dislocations in the lattice of dislocations and so on.

We see that a sophisticated hierarchical structure is realized in the system under consideration. There is an infinite number of "particle" generations, the "particles" of $m$ th generation being dislocations in the lattice of the particles of $m-1$ generation. The "particle" density for each generation is given by [8]

$$
\begin{gathered}
n_{m}=-\beta \frac{d}{d x} \sqrt{n_{m-1}}=\frac{A_{m}}{\beta^{2} x^{2}}\left(\frac{\beta^{2} b}{x}\right)^{2-2^{-m}}, \\
A_{m}=\prod_{j=1}^{m}\left(1-2^{-j}\right)^{2^{j-m}}
\end{gathered}
$$

The densities of high generation particles quickly approach the limiting function $n_{\infty}=\beta^{2} / x^{2}$. For the limiting distribution, the notion of lattice no longer makes sense since the average interparticle distance is of the order of $x$. This also implies that the difference between generations becomes less pronounced with growing $m$. Owing to this, only a finite number of generations can be actually observed in a realistic system. This depends on the system size: the larger the size, the more generations can be resolved. The estimations for a Quantum Hall bar show that at realistic submillimeter scale one can see a lattice formed by forth generation "particles" and possibly distinct "particles" of fifth generation.

A small segment of the resulting arrangement is presented in Fig.2. It is an illustration, no attempt to minimize the structure energy has been performed. For simplicity I assumed that the dislocations shift the particles in $y$ direction only. The magnitude of the shift is proportional to the bearing at which the particle is seen from the dislocation location. 
Three generations are visible in Fig. 2: quasiholes, dislocations in quasihole lattice and a dislocation in the dislocation lattice.

What may be the experimental manifestations of the hierarchical crystal? Since the quasiparticles forming the structure are localized they do not contribute to electric current and hardly can be seen in dc transport measurements that are so common in Quantum Hall devices. It seem feasible [9] to measure directly the charge distribution over the bar by highly sensitive Coulomb blockade electrometer. Another possibility is to study microwave response of the heterostructure: the oscillatory modes of the hierarchical crystal can be revealed in this way, similar to those ones of a common Wigner crystal. [10]

The arguments given are so evident and general that the scheme proposed shall not be supported by any analytical or numerical calculations. Besides, such calculations are very difficult to perform so it remains a challenging task for the future.

There is an intrinsic difficulty with numericals arising from the simple fact that any arrangement of dislocations corresponds to a local energy minimum. Thus they are difficult to anneal, and the absolute minimum is difficult to find. The state of the art of the Wigner numerics one can find in [11,5]. Due to the difficulties described, the number of particles considered is at most of the order of hundred. It makes it very difficult to get rid of edge effects. Nevertheless, the very beginning of the hierarchical scenario can bee seen from these numerical results. Figures $4 \mathrm{a}, 4 \mathrm{~b}$ of 5 nicely demonstrate how the dislocations appear in the structure.

I believe that such calculations are worth doing with much greater number of particles, despite enormous CPU time required. The reason is a general nature of the hierarchical ordering described. The above reasoning can be applied to any system of classical particles with repulsion provided the external forces fix their average density. There are various physical realizations of this such as Abrikosov vortices in superconducting film subjected to external magnetic field, charged dopants in semiconductors placed in external nonuniform electric field, electrons on liquid helium surface in nonuniform field and others. So I conclude stressing that the hierarchical ordering may occur in many instances.

I am indebted to L. S. Levitov, L.S. Glazman and many others for very instructive discussions of the results. This work is a part of the research program of the "Stichting voor Fundamenteel Onderzoek der Materie" (FOM), which is financially supported from the "Nederlandse Organisatie voor Wetenschappelijk Onderzoek" (NWO). 


\section{REFERENCES}

[1] T. Chakraborty and P. Pietiläinen, The fractional quantum Hall effect, Springer-Verlag, Berlin Heidelberg 1988.

[2] J. Dempsey, B. Y. Gelfand, and B. I. Halperin, Phys. Rev. Lett. 70, 3639 (1993); D. J. Thouless, Phys. Rev. Lett. 71, 1879 (1993); N. R. Cooper and J. T. Chalker, Phys. Rev. B 48, 4530 (1993)

[3] P. L. McEuen, E. B. Foxman, Jari Kinaret, U. Meirav, M. A. Kastner, N. S. Wingreen and S. J. Wind, Phys. Rev. B 45,11419 (1992).

[4] D. B. Chklovskii, B. I. Shklovskii, and L. I. Glazman, Phys. Rev. B 46, 4026 (1992).

[5] H. A. Fertig and R. Côté, Phys. Rev. B , 48, 2391 (1993).

[6] Yu. V. Nazarov and A. V. Khaetskii, Phys. Rev. B 49, 5077 (1994).

[7] J. R. Barber, Elasticity (Kluwer, Dordrecht, 1992).

[8] Here I assumed that all generations form a triangular lattice. If this is not the case, $\beta$ 's will be different for different for different generations albeit the power law exponents remain the same.

[9] H. Pothier, private communication.

[10] E. Y. Andrei, G. Deville, D.C. Glattli, and F. I. B. Williams, Phys. Rev. Lett. 60, 2765 (1988).

[11] H. A. Fertig, R. Côté, A. H. MacDonald and S. Das Sarma, Phys. Rev. Lett. 69, 816 (1992); H. A. Fertig and S. Das Sarma, Phys. Rev. B 47, 10484 (1993). 


\section{FIGURES}

FIG. 1. The structure of a Quantum Hall bar edge. The density profile is presented. 2D gas

can be schematically subdivided into two regions: metallic state (i), incompressible state doped by quasiholes (ii).

FIG. 2. The segment of a hierarchical crystal. Dots correspond to quasiholes. Dislocations

in quasihole lattice are marked by diamonds. The cross marks the dislocation in the dislocation lattice: the second generation "particle". 